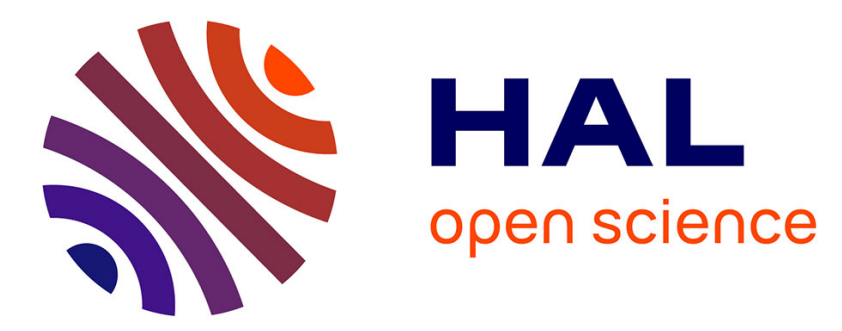

\title{
La doctrine romaine de l'inspiration de Léon XIII à Benoît XV (1893-1920) : la production d'une nouvelle orthodoxie
}

Francesco Beretta

\section{- To cite this version:}

Francesco Beretta. La doctrine romaine de l'inspiration de Léon XIII à Benoît XV (1893-1920) : la production d'une nouvelle orthodoxie. Autour d'un petit livre. Alfred Loisy cent ans après, May 2003, Paris, France. pp.47-60. halshs-00004689

\section{HAL Id: halshs-00004689 \\ https://shs.hal.science/halshs-00004689}

Submitted on 22 Sep 2005

HAL is a multi-disciplinary open access archive for the deposit and dissemination of scientific research documents, whether they are published or not. The documents may come from teaching and research institutions in France or abroad, or from public or private research centers.
L'archive ouverte pluridisciplinaire $\mathbf{H A L}$, est destinée au dépôt et à la diffusion de documents scientifiques de niveau recherche, publiés ou non, émanant des établissements d'enseignement et de recherche français ou étrangers, des laboratoires publics ou privés. 
La doctrine romaine de l'inspiration de Léon XIII à Benoît XV (1893-1920) :

la production d'une nouvelle orthodoxie

\author{
par \\ Francesco Beretta \\ (CNRS - LARHRA UMR 5190, Lyon)
}

Dans la conclusion de son ouvrage fondateur pour l'histoire critique du modernisme catholique, Émile Poulat soulève le problème de l'insertion de l'œuvre d'Alfred Loisy à l'intérieur de la "transformation mentale collective" qu'elle a elle-même contribué à produire. L'intention des ouvrages de Loisy, de ses "petits livres", est celle d"“ occuper des positions différentes des positions traditionnelles, de découvrir les voies nouvelles dans lesquelles l'orthodoxie pourrait se reconnaître "1. Expression d'une différence radicale d'idées, plus encore de "manières de penser" et de pratiques savantes, cette transformation pose le problème de la définition de l'orthodoxie, de la "doctrine-étalon "2 par rapport à laquelle il s'agit de prendre la mesure des positions de Loisy, ou de celles de tout autre auteur à une époque donnée.

Comment cerner l'orthodoxie en vigueur? Dans quels textes en trouver l'expression? Quelle signification attribuer à la doctrine propre à ces textes : s'agit-il d'une position traditionnelle ou d'une nouvelle orthodoxie? Et encore : l'orthodoxie de 1903 est-elle encore en vigueur aujourd'hui? La " transformation mentale collective" inaugurée à l'époque de l'activité intellectuelle de Loisy n'a-t-elle pas amené, à travers un siècle, à la production d'une nouvelle orthodoxie?

Il s'agit d'un problème de taille, dont il faut être conscient si on veut déjouer un piège classique en histoire intellectuelle, surtout pour la période contemporaine : utiliser ses propres représentations, souvent inconscientes, comme mesure de l'orthodoxie des conceptions doctrinales qu'on se propose d'étudier. "Chacun suit son idée pour la faire avérer"3. Tel est le cas, par exemple, du long article du Dictionnaire de théologie catholique consacré par Eugène Mangenot à la question de la nature et des effets de l'inspiration biblique —comme on disait à l'époque-, dont le principal serait l'inerrance ${ }^{4}$. Dans ce texte, rédigé au début des années 1920, l'auteur présente en détail

1 É. Poulat, Histoire, dogme et critique dans la crise moderniste, 3e éd., Paris, Albin Michel, 1996, pp. 616-618. Cf. “ Avant-propos à la 3e édition ”, en particulier pp. XXXIV-XLIX.

2 Ibid., p. 620.

3 Ibid.

4 E. Mangenot, “Inspiration de l'Écriture ”, Dictionnaire de théologie catholique, t.7, 2e partie, Paris, Letouzey et Ané, 1923, coll. 2068-2266. 
les débats qui ont eu lieu au sujet de la vérité biblique au cours des quatre décennies qui précèdent. Les positions des différents exégètes et théologiens sont mesurées, voir même reconstituées à la lumière de la doctrine des deux principales interventions du magistère pontifical à ce sujet : les encycliques Providentissimus Deus de Léon XIII, en 1893, et Spiritus paraclitus de Benoît XV, en $1920^{5}$.

Tel est également le cas d'études plus récentes, et notamment de celles publiées à l'occasion du centenaire de la publication de l'encyclique Providentissimus Deus. Ces études semblent considérer que l'orthodoxie en matière d'inerrance biblique a été fixée par Léon XIII, alors que la doctrine de Vatican II à ce sujet, mais surtout les orientations actuelles en matière de vérité biblique, se situent en position antinomique par rapport à celle de l'encyclique de $1893^{6}$. Quelle est donc l'orthodoxie catholique en matière d'inerrance biblique?

Tout en laissant aux théologiens la réponse théorique à cette question, j'aimerais proposer ici — dans le prolongement de l'analyse critique d'Émile Poulat— quelques jalons en vue d'une reconstitution des mécanismes de production d'orthodoxie dans l'espace intellectuel catholique au tournant du XXe siècle, dont on trouvera de plus amples développements dans un ouvrage à paraître prochainement ${ }^{7}$. À cette fin, j'étudierai la fonction des deux encycliques de 1893 et de 1920 en tant qu'instruments de production d'une nouvelle orthodoxie en matière d'exégèse, grâce à l'imposition officielle d'une doctrine particulière de l'inspiration et de la vérité intégrale de la Bible. Je montrerai en même temps pourquoi la doctrine de l'inspiration et, surtout, celle de l'inerrance biblique est décisive pour la question de l'historicité, et donc de l'interprétation des premiers chapitres de la Genèse. L'orthodoxie de la méthode exégétique est indissociablement liée, à l'époque en question, à l'orthodoxie de la doctrine de l'inspiration ${ }^{8}$.

\section{Champ théologique et production d'orthodoxie}

Quelques considérations préalables s'imposent en vue d'expliciter la problématique utilisée dans la construction de l'objet retenu ici. J'appliquerai l'analyse des champs de

5 Pour le texte des encycliques, voir Enchiridion biblicum (=EB), Bologna, Edizioni Dehoniane, 1993, respectivement nn. 81-134 et 440-495.

6 Pour une analyse plus développée de cette question, voir mon étude “ De l'inerrance absolue à la vérité salvifique de l'Écriture: l'encyclique Providentissimus Deus (1893) entre Vatican I et Vatican II ", Freiburger Zeitschrift für Philosophie und Theologie, XLVI, 1999, pp. 461-501, et, pour la discussion contemporaine, H. Gabel, Inspirationsverständnis im Wandel, Mainz, Grünewald, 1991 et F. Martin, Pour une théologie de la lettre, Paris, Cerf, 1996.

7 F. Beretta, Le combat pour une nouvelle orthodoxie, à paraître aux éditions Les Belles Lettres.

8 Cf. Poulat, op. cit., pp. LVII-LIX. 
production culturelle développée par Pierre Bourdieu, et en particulier celle du champ scientifique $^{9}$, à la reconstitution $\mathrm{du}$ fonctionnement du champ théologique. Cette approche permet d'historiciser le problème de la production de l'orthodoxie catholique et d'éviter les pièges indiqués ci-dessus, dont le principal consiste à croire -en adoptant ainsi le point de vue des agents du champ étudié- qu'il existe une continuité de fond dans l'orthodoxie et que celle-ci est définissable dans l'abstrait.

Du point de vue de l'historien, l'orthodoxie est, à une époque donnée, le produit des rapports de forces et des luttes à l'intérieur du champ théologique. Elle est le résultat de la concurrence entre agents qui soutiennent des positions différentes, parfois antinomiques et irréductibles, et qui s'efforcent de diffuser leurs positions, voire de les imposer aux autres agents. L'orthodoxie n'est donc pas présupposée, elle est un produit; elle n'est pas une et uniforme, mais complexe et soumise à un processus continuel d'évolution et de transformation. Comme l'orthodoxie n'est rien d'autre que ce que les agents considèrent, à un moment donné, comme étant orthodoxe, elle est l'expression de la configuration du champ théologique à ce moment-là.

Une telle approche soulève le problème de l'articulation entre champ théologique et champ religieux, le deuxième étant à bien distinguer du premier. Aussi, se pose la question de la situation de ces champs par rapport à l'espace social, car l'orthodoxie n'existe pas indépendamment de la société, ou du groupe social, dont elle forge, et exprime, les représentations. Mais ces considérations sont trop complexes pour être abordées dans ce cadre. Retenons seulement que ce qui est en jeu dans la production d'orthodoxie telle qu'elle est envisagée ici, ce ne sont pas les croyances qui fondent la vie sociale mais les stratégies d'argumentation rationnelle, de construction méthodique d'objets, de validation de la connaissance qui fondent la conception de la théologie comme science.

Un autre problème concerne la réalité dont s'occupe cette science, la Révélation chrétienne, dont la nature est différente de celle des objets propres aux disciplines qualifiées, à notre époque, de sciences: le monde physique, biologique et social. Toutefois, l'historien ne s'intéresse pas -à la différence du théologien ou du philosophe - à la discussion du postulat ontologique qui fonde l'objet de la science théologique, mais il se propose d'étudier les règles de cette rationalité réfléchie, ses institutions de production et de régulation intellectuelle, les pratiques savantes et les conflits entre dominants et dominés qu'elle engendre.

On reconnaît ainsi une homologie entre champ scientifique et champ théologique qui permet d'appliquer au deuxième les outils développés pour l'analyse du premier et

9 Pour une synthèse des éléments essentiels de l'analyse du champ scientifique, voir P. Bourdieu, Science de la science et réflexivité, Paris, Éditions Raisons d'agir, 2001. Cf. L. Pinto, Pierre Bourdieu et la théorie du monde social, Paris, Albin Michel, 1998. 
qui se fonde sur l'autonomie - inévitablement relative mais non moins effective - du champ théologique. Cette autonomie se construit autour de la figure du théologien, formé et reconnu comme tel par ses pairs, qui dispose donc d'un habitus disciplinaire qui le distingue structurellement des autres agents du champ religieux, et notamment des évêques ${ }^{10}$.

À ce sujet, il faut relever une particularité du champ théologique : si seuls les théologiens appartiennent, légitimement, au champ, la réalité qui est objet de leur science, la Révélation chrétienne, est un discours fixé, en même temps, par leur propre science et par des instances de régulation intellectuelle qui interviennent d'autorité : les Conciles et le pape; dans une moindre mesure, les évêques et les supérieurs d'Ordres religieux. L'orthodoxie, entendue au sens large d'opinion droite, se situe donc à l'intersection entre champ théologique et champ religieux, elle doit être recevable par le groupe social de référence - la communauté croyante - et elle est indispensable pour appartenir aux deux champs.

Les instances de régulation intellectuelle, et notamment les deux principales que sont les Conciles et le Siège apostolique, ne sont toutefois pas purement externes au champ théologique car les experts qui produisent conceptuellement les prises de position officielles en matière doctrinale sont eux-mêmes des agents du champ théologique. Ils peuvent donc profiter du pouvoir dont ils disposent à l'intérieur des institutions de régulation intellectuelle pour affirmer leurs propres conceptions ${ }^{11}$. Le champ théologique se structure ainsi autour des institutions de production intellectuelle — séminaires, Facultés de théologie, cercles savants, congrès catholiques- et des institutions de régulation intellectuelle - le pape et les Congrégations romaines, les Conciles, à certaines époques les Facultés elles-mêmes. Pour étudier les mécanismes de production d'orthodoxie, il est donc indispensable de reconstituer le fonctionnement du champ théologique et de situer les agents, et leurs positions, à l'intérieur de celui-ci' ${ }^{12}$.

Dans l'espace intellectuel catholique de la fin du XIXe et du début du XXe siècle, les encycliques revêtent une importance particulière dans la production d'orthodoxie en raison de la très grande valeur que la quasi-totalité des agents attribuent à ces

10 P. Bourdieu et M. de Saint-Martin, “La Sainte Famille. L’épiscopat français dans le champ du pouvoir”, Actes de la recherche en sciences sociales, (44-45), 1982, pp. 2-53 : 23-29. Cf. E. Dianteill, “Pierre Bourdieu et la religion. Synthèse critique d'une synthèse critique ”, Archives de sciences sociales des religions, XLVII (118), 2002, pp. 5-19.

11 P. Colin, L'audace et le soupçon, Paris, Desclée de Brouwer, 1997, a bien mis en évidence ce phénomène, cf. en particulier p. 508.

12 Cf. F. Beretta, "La Congrégation de l'Inquisition et la censure doctrinale: affermissement du pouvoir d'une institution de régulation intellectuelle ", dans les Actes du colloque Inquisition et Pouvoir (Aix-en-Provence 2002, sous presse), pp. 41-54. 
documents, indépendamment de leur propre situation intellectuelle par rapport aux positions exprimées par ces textes. À ce propos il faut distinguer un double phénomène de production d'orthodoxie.

D'une part, il y a production d'orthodoxie lors de la rédaction des encycliques. Pour saisir la signification de ces textes, il faut analyser leur visée doctrinale précise en étudiant les écrits des experts qui les ont rédigés, ainsi que les commentaires officieux que ces mêmes experts font paraître après la publication des encycliques pour en expliquer le sens. En même temps, il faut étudier comment la doctrine propre aux experts, et que la caution pontificale transforme en doctrine officielle, se situe par rapport aux autres positions présentes dans le champ, et qui se distinguent, ou s'opposent à la première.

Mais, d'autre part, la production d'orthodoxie se fait aussi, peut-être même principalement, par la réception des textes des encycliques. L'interprétation de leur doctrine peut être conforme aux intentions des rédacteurs mais elle peut aussi aller jusqu'à en détourner complètement le sens. Ceci s'explique par la nécessité de la part des agents qui soutiennent des positions antinomiques par rapport à celles des rédacteurs, de se soumettre formellement aux directives pontificales - condition indispensable à l'appartenance au champ théologique - mais, en même temps, à la volonté d'affirmer, dans la mesure du possible, des positions différentes. Les agents appartenant au camp qui a produit la doctrine officielle, et qui contrôlent les institutions de régulation intellectuelle dans le champ, interviendront pour rectifier et préciser l'interprétation de ces textes fondateurs d'orthodoxie.

Passons maintenant à l'analyse de la production d'une nouvelle orthodoxie par les deux encycliques en question, en matière d'inerrance biblique et d'interprétation de l'Écriture sainte. Pour saisir la signification de l'encyclique Providentissimus Deus, il est indispensable de remonter au texte qui la précède en tant que document fondateur d'orthodoxie, c'est-à-dire à la constitution Dei Filius du Concile Vatican I. En effet, comme nous le verrons, l'encyclique de 1893 représente une interprétation officielle du deuxième chapitre de la constitution Dei Filius, promulguée en 1870 et consacrée à la "Foi catholique", chapitre qui concerne la Révélation et, plus particulièrement, l'inspiration et l'interprétation de l'Écriture.

\section{La doctrine de Vatican I et sa réception}

Comme l'indiquent les annotations du schéma préparatoire révisé par la commission doctrinale, Vatican I se limite à proposer l'enseignement concernant l'inspiration qu'on trouve déjà dans les décrets des Conciles de Florence et de Trente : “L'Église tient [les livres de l'Ancien et du Nouveau Testament] pour [sacrés et canoniques] [...] parce que écrits sous l'inspiration du Saint-Esprit, ils ont Dieu pour 
auteur et ont été transmis comme tels à l'Église "13. Le texte promulgué par le Concile se trouve en retrait par rapport à la doctrine qu'avait formulée dans le schéma préparatoire le célèbre théologien jésuite Jean-Baptiste Franzelin, l'un des principaux représentants de l'école théologique des jésuites qui enseignent à l'Université Grégorienne et que, pour simplifier, nous appellerons l'École romaine ${ }^{14}$.

Quant à la nature de l'inspiration, Franzelin adopte la doctrine dite de l'inspiration réelle. Il pense donc que l'inspiration concerne non pas les mots de l'Écriture, comme le veut la doctrine de l'inspiration verbale, mais les assertions qui se trouvent dans la Bible, les “ res et sententiæ". Quant à l'étendue de l'inspiration, le théologien jésuite pense que toutes les assertions de l'hagiographe sont à considérer comme divinement inspirées, mêmes celles qui concernent l'histoire ou le détail des récits. De cette conception, qui insiste sur la causalité efficiente de l'inspiration découle l'inerrance intégrale de la Bible, car Dieu, vérité première, ne peut pas mentir. Dans le schéma préparatoire, Franzelin parle donc d'une autorité littéraire de Dieu, attribuant à Dieu lui-même, en premier lieu, la rédaction de l'Écriture ${ }^{15}$.

Les interventions des Pères de la minorité à Vatican I - les évêques Dupanloup, Ginoulhiac, Ketteler et surtout Meignan, évêque de Châlons-sur-Marne- amèneront à la révision du texte qui reprend la formulation plus sobre des Conciles précédents en parlant de Dieu simplement comme auteur de l'Écriture, sans préciser la modalité de cette autorité divine dont la définition est laissée explicitement à la libre discussion des écoles théologiques. À ce propos, il faut remarquer que les évêques de la minorité se sont opposés à plusieurs reprises à la tentative des jésuites de l'École romaine de faire entrer leur propre doctrine dans les textes du Concile en la présentant comme celle de la Tradition. Ainsi, l'évêque de Mayence Ketteler souligne qu'il est de coutume dans l'Église de condamner les hérésies et non pas de trancher les questions d'école. La formulation retenue dans la constitution Dei Filius évite donc explicitement de trancher la question de la nature et de l'étendue de l'inspiration, en la laissant à la discussion des écoles théologiques ${ }^{16}$.

Les problèmes posés à l'exégèse traditionnelle par le développement, surtout dès le milieu du XIXe siècle, de la critique biblique, de l'orientalisme et de l'histoire comparée des religions, amèneront plusieurs auteurs catholiques à chercher une

13 Les Conciles cecuméniques, t.II/2: Les décrets. Trente à Vatican II, Paris, Cerf, 1994, p. 1639 (souligné par moi).

14 Cf. K. H. Neufeld, "La scuola romana", R. Fisichella (éd.), Storia della teologia, t. 3, Roma/Bologna, Edizioni Dehoniane, 1996, pp. 267-283.

15 Cf. Beretta, “ De l'inerrance absolue ”, pp. 464-467.

16 Ibid., pp. 471-476. 
nouvelle définition de l'inerrance de l'Écriture ${ }^{17}$. L'analyse des débats et des polémiques au cours des années 1880 montre qu'il s'agit d'un combat pour l'interprétation orthodoxe de la formule retenue dans le texte promulgué au concile Vatican I, et que ce combat voit s'opposer d'une part les théologiens intransigeants, avec l'appui de quelques exégètes appartenant à la même école — tel le sulpicien Vigouroux, auteur d'un Manuel biblique classique, dont nous reparlerons-, et d'autre part les exégètes et théologiens catholiques ouverts à l'application à la Bible des nouvelles méthodes développées pour l'étude des textes de l'Antiquité.

\section{La "Question biblique "}

Lorsque le recteur de l'Institut catholique de Paris, Mgr d'Hulst, dans son célèbre article de 1893 publié dans le Correspondant ${ }^{18}$, parle de l'opposition entre une "école étroite" et une "école large" 19 , il ne fait que tenter de s'opposer à la pression croissante exercée par les tenants de l'École romaine -représentée en France en particulier par le jésuite Joseph Brucker- en invoquant le principe traditionnel de la liberté de discussion entre écoles théologiques. Comme Mgr d'Hulst l'écrit en 1892, dans une note qui lui servira de base pour rédiger le célèbre article, la position de l'école étroite est claire : Dieu est " rendu responsable de la vérité intrinsèque de tout ce qui est affirmé dans la Bible, chaque fois qu'il n'est pas possible de prêter à l'auteur sacré une intention allégorique". Cette conception - qui est celle du cardinal Franzelin - est défendue au nom de la Tradition dont les théologiens affirment être les témoins ${ }^{20}$.

De l'autre côté, continue Mgr d'Hulst, les “exégètes et critiques catholiques” maintiennent le fait de l'inspiration de l'Écriture, défini par les Conciles, mais ils reconnaissent en même temps 1 "“ inexactitude" au point de vue historique de certains passages de la Bible. Ils ne croient donc plus possible de soutenir "la doctrine de l'inerrance absolue en matière historique, de l'inerrance qui devrait exclure jusqu'aux

17 À ce sujet, voir F. Laplanche, La Bible en France entre mythe et critique (XVIe-XIXe siècle), Paris, Albin Michel, 1994, pp. 187-206 et, du même auteur, “La question biblique au temps de Monseigneur d'Hulst", C. Bressolette (éd.), Monseigneur d'Hulst fondateur de l'Institut catholique de Paris, Paris, Beauchesne, 1998, pp. 137-156, ainsi que J. Beumer, Die katholische Inspirationslehre zwischen Vatikanum I und II, Stuttgart, Verlag Katholisches Bibelwerk, 1967 et J. T. Burtchaell, Catholic Theories of Biblical Inspiration since 1810. A Review and Critique, Cambridge, Cambridge UP, 1969.

18 M. d'Hulst, “La question biblique ”, Le Correspondant, CLXX, 1893, pp. 201-251.

19 L'usage de ces termes s'établit dès le milieu des annés 1880 comme le montre, entre autres, un article de J. Brucker publié en janvier 1888, “Questions actuelles d'exégèse et d'apologie biblique ", Études, XXV, 1888, p. 85 sq.

20 F. Beretta, Monseigneur d'Hulst et la science chrétienne, Paris, Beauchesne, 1996, p. 376 sq. 
lapsus de mémoire, jusqu'aux anachronismes" et ils demandent qu'on entende "plus largement les effets de l'inspiration "21. D'où le nom d'école large.

Cette conception avait déjà été soutenue par Mgr Meignan, dans un ouvrage publié en 1864 lorsqu'il était professeur d'Écriture sainte à la Faculté de théologie de la Sorbonne ${ }^{22}$. Elle est formulée encore plus clairement, en 1880, dans Les Origines de l'histoire de François Lenormant, orientaliste catholique élu à l'Académie des Inscriptions en 1881. Dans son ouvrage, Lenormant étudie les relations qui existent entre le récit biblique et ceux issus des littératures de l'Orient ancien, dont les éditions se multiplient dès le milieu du XIXe siècle. Dans une démarche qui relève de l'histoire comparée des religions, Lenormant reconnaît l'origine légendaire, voire mythologique des récits qu'on trouve dans les premiers chapitres de la Genèse et qui retracent les origines de l'humanité. Ceux-ci ne relèvent pas, selon lui, du genre historique, comme l'avaient soutenu jusque-là les exégètes catholiques. En vertu de l'inspiration divine, ces récits repris des littératures de l'Orient ancien auraient été purifiés des éléments polythéistes pour les adapter aux croyances religieuses du peuple hébreu ${ }^{23}$.

Le principe commun à des auteurs comme Meignan et Lenormant, et qui sera également formulé par le cardinal Newman dans un article publié, en traduction, dans le Correspondant de 1884, est formulé avec clarté par un confrère du P. Brucker qui, en décembre 1883, rapporte à ce dernier les doctrines dont il a entendu parler à Paris, probablement de la bouche même du recteur de l'Institut catholique d'Hulst :

pour savoir ce qui est proprement inspiré, il faut consulter le but que Dieu avait en vue, le salut des hommes : de là tout ce qui ne se rapporte pas directement à la foi et aux mœurs, nécessaires au salut, est en dehors de l'inspiration ${ }^{24}$.

Brucker publiera plusieurs articles pour réfuter ces opinions et réaffirmer la doctrine de son école qu'il considère, à la suite du cardinal Franzelin, comme appartenant à la Tradition.

À ce stade de ma reconstitution, il importe de relever deux éléments. Premièrement, les positions de l'école étroite et celles de l'école large sont irréductibles. Car si Franzelin et ses partisans définissent l'inerrance de l'Écriture à partir de la causalité efficiente de l'inspiration, en faisant de Dieu l'auteur de toutes les assertions de l'hagiographe, leurs adversaires conçoivent l'inspiration à partir de la cause finale : ce qui compte est le but qu'avait Dieu en inspirant l'hagiographe, ce but étant le Salut

21 Ibid., pp. 377-381 (souligné par moi).

22 Beretta, “De l'inerrance absolue ”, p. 468.

23 F. Lenormant, Les origines de l'histoire d'après la Bible et les traditions des peuples orientaux, t.1, Paris, Maisonneuve, 1880, p. XVIII sq. Ces principes sont fomulés de façon encore plus explicite dans la première partie du deuxième tome, Paris, Maisonneuve, 2e éd. 1882, pp. 265-269, note 1.

24 Beretta, Monseigneur d'Hulst, p. 378, note 1. 
chrétien. Toutes les assertions bibliques qui se rapportent à cette fin - la cause finalesont vraies, les autres sont humaines, donc elles peuvent être inexactes.

Deuxièmement, la fonction de ces deux conceptions irréductibles de l'inerrance biblique est celle de légitimer deux pratiques radicalement différentes de l'exégèse. Pour les tenants de l'école étroite, il s'agit de défendre une lecture littéraliste de la Bible et, tout particulièrement, celle des premiers chapitres de la Genèse. Car sur cette lecture, centrée sur la véracité historique du récit de la création d'Adam et d'Ève, et de leur péché, se construit tout l'édifice de la théologie scolastique ${ }^{25}$. En revanche, pour les tenants de l'école large, qu'ils soient exégètes, orientalistes ou théologiens ouverts aux acquis de ces disciplines, l'acceptation des résultats de la méthode critique et de l'histoire des religions - qui invitent à considérer que le récit de la Création n'appartient pas au genre littéraire historique - implique une redéfinition de la doctrine de l'inspiration et, en réalité, une refondation de la théologie elle-même, car le sens de l'Écriture produit par les nouvelles disciplines n'est pas le même que celui de la méthode scolastique.

Un témoignage très lucide sur cette situation nous est donné dans la leçon d'ouverture du cours d'Écriture sainte de 1892, que tient à l'Institut catholique de Paris un jeune exégète très prometteur. Choisi par son évêque, Mgr Meignan, pour continuer les études, il est protégé par le recteur d'Hulst pour en faire " l'exégète de la maison "26. Il s'agit, évidemment, d'Alfred Loisy.

Dans le texte de son cours, publié dans son éphémère revue L'enseignement biblique, Loisy fait la distinction entre un "sens doctrinal" de l'Écriture, "établi par l'autorité de l'Église ou l'enseignement unanime des Pères", et un "sens historique", produit par une interprétation "purement historique et critique des Livres saints". Loisy souligne la différence importante qui subsiste entre ces deux sens de l'Écriture, produits par deux méthodes radicalement différentes. Car si les "théologiens scolastiques " tirent du troisième chapitre de la Genèse une théorie du péché originel et de la rédemption aussi complète [...] que celle du concile de Trente", l'exégète "critique" "n'est pas tenu de trouver [dans le sens vu par l'auteur biblique] toute l'ampleur du commentaire traditionnel "27. Quant à la doctrine de l'inspiration, Loisy semble évoluer, à cette époque, d'une position concernant la vérité de l'Écriture proche de celle de Lenormant —admettant une transformation, dans un sens monothéiste, des

25 Voir, par exemple, le traité de théologie dogmatique du secrétaire de la Commission biblique L. Janssens, Summa theologica ad modum commentarii in Aquiinatis summam prosentis avi studiis aptatam, t. 8, Roma, Typis Polyglottis Vaticanis, 1909, pp. 1 et 388.

26 A. Loisy, Mémoires, t.I, Paris, É. Nourry, 1930, p. 179.

27 A. Loisy, “De la critique biblique ”, L’Enseignement biblique, I, 1892, fascicule séparé, p. 13 sq. 
récits mythologiques de l'Orient ancien — vers la conception de la vérité de l'Écriture relative ou "proportionnée" au temps de sa rédaction et au progrès doctrinal, qui lui sera propre $^{28}$.

L'affrontement se fait donc sur des positions irréductibles tant au point de vue de la méthode exégétique qu'à celui de la doctrine de l'inspiration et de l'inerrance, la deuxième étant appelée à fournir sa légitimation théorique à la première. La crise est aiguë au début de 1893, car le supérieur de Saint-Sulpice, Monsieur Icard, a jeté l'interdit sur le cours de Loisy et les tenants de l'école étroite ne cessent de l'attaquer plus ou moins ouvertement. D'Hulst décide de mettre en jeu son autorité de recteur et publie dans le Correspondant, en janvier 1893, son célèbre article intitulé " La question biblique ", dans lequel il va jusqu'à défier l'autorité des Congrégations romaines en citant longuement le livre de Lenormant qui a été mis à l'Index en $1887^{29}$.

Retenons de cet article deux éléments essentiels. Premièrement, le fait de parler de deux écoles représente une prise de position claire -et percevable comme telle à l'époque - en faveur de la liberté de discussion. Toutefois, les champions de l'école étroite refuseront d'admettre cette liberté et ils profiteront de leur pouvoir croissant dans la Curie romaine pour obtenir du pape Léon XIII une condamnation formelle de la doctrine de 1'“ école large", telle qu'elle est exposée par le recteur de l'Institut catholique de Paris dans son article ${ }^{30}$. Deuxièmement, cette doctrine se rapproche davantage de celle de Lenormant et de Loisy, que de celle de Newman, et elle n'est pas sans originalité. Ce qui est restreint, selon d'Hulst, ce n'est pas l'inspiration, mais uniquement l'inerrance : tout est inspiré, même la lettre, mais seulement à un certain point de vue, le point de vue religieux. Ce qui ne se rapporte pas à cette fin de l'inspiration ne relève que de l'auteur humain et, de ce fait, il peut y avoir des “ énoncés inexacts dans la Bible ”. L’inerrance absolue, même dans les détails historiques, n'est plus soutenable 31 .

\section{L'encyclique de Léon XIII et sa réception}

La doctrine exposée dans l'article de Mgr d'Hulst sera condamnée formellement

28 A. Loisy, Compte rendu d'un ouvrage de P. Dausch, L'Enseignement biblique, I(3), 1892, pp. 514. Cf. Poulat, op. cit., pp. 81-83, 169 et R. Ciappa, Storia e teologia. L'itinerario intellettuale di Alfred Loisy (1883-1903), Napoli, Liguori, 1993, pp. 17-30, 72-80 et 167-171.

29 M. d'Hulst, “La question biblique”, pp. 228-231. Cf. F. Beretta, “Dalla messa all'Indice di Lenormant all'enciclica Providentissimus Deus (1887-1893): il magistero romano di fronte alla Question biblique", L'Inquisizione e gli storici: un cantiere aperto, Roma, Accademia nazionale dei Lincei, 2000, pp. 245-260: 252-254.

30 Beretta, Monseigneur d'Hulst, pp. 118-122.

31 D’Hulst, op. cit., pp. 220-233. 
par l'encyclique Providentissimus Deus, publiée fin novembre 1893. Une version préparatoire, rédigée par l'exégète jésuite Cornély, a été substantiellement transformée au point de vue doctrinal, sous l'influence du cardinal jésuite Mazzella, représentant très puissant de l'École romaine. Voici le passage essentiel de l'encyclique de Léon XIII :

mais il sera toujours absolument interdit, soit de restreindre l'inspiration à certaines parties seulement de la sainte Écriture, soit de concéder que l'auteur sacré lui-même s'est trompé. On ne saurait, en effet, tolérer le système de ceux qui, pour échapper à ces difficultés, ne craignent pas d'admettre que l'inspiration divine s'applique aux choses de la foi et des mœurs, mais à rien de plus, parce qu'ils croient faussement que s'il s'agit de la vérité des textes, on ne doit pas tant rechercher ce que Dieu a dit, qu'examiner pour quel motif il l'a dit ${ }^{32}$.

La conception de la causalité finale de la vérité biblique, soutenue sous différentes formes par l'école large, a été ainsi formellement réprouvée par l'encyclique et déclarée même comme étant incompatible avec la foi ${ }^{33}$.

En effet, selon Léon XIII la vraie doctrine catholique de l'inspiration est celle de la causalité efficiente, qui attribue à Dieu l'autorité de toutes les assertions de l'hagiographe. Le pape affirme ainsi l'inerrance absolue de l'Écriture, même en matière historique $^{34}$. De plus, l'encyclique de 1893 impose aux catholiques de pratiquer la méthode scolastique: dans la recherche du sens de l'Écriture, l'exégète est ainsi tenu d'utiliser principalement le texte latin de la Vulgate, et non pas les versions originales hébraïque et grecque, et d'“établir par son enseignement, que seule l'interprétation [fixée par l'Église] peut être justifiée par les lois d'une saine herméneutique "35.

L'encyclique Providentissimus Deus représente donc un moment important de production d'orthodoxie. Les tenants de l'école étroite, qui, pour des raisons politiques et intellectuelles, contrôlent non seulement les Congrégations romaines, mais encore la parole pontificale elle-même, imposent leur propre position en la présentant comme la foi de l'Église. Ils arrivent ainsi à obtenir, dans l'encyclique de 1893, une victoire qu'ils n'avaient même pas pu espérer à Vatican I, en produisant, grâce à cette prise de position pontificale, un nouveau cadre doctrinal officiel de l'activité des exégètes catholiques.

Mais cette victoire devait être consolidée. Car si les tenants de la position adverse s'étaient soumis formellement, en 1893, aux indications pontificales, ils avaient continué à pratiquer une méthode exégétique incompatible avec celle de la scolastique. De plus, pour légitimer leur pratique d'exégètes, ils s'étaient efforcés d'introduire des principes herméneutiques respectant, en apparence, l'enseignement de Providentissimus

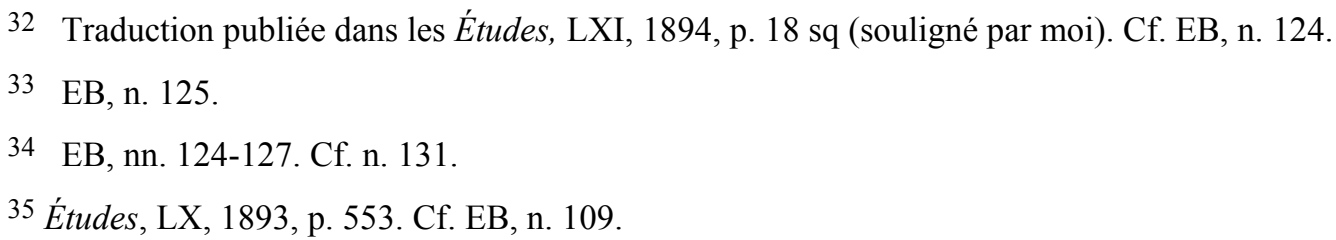


Deus. On les voit ainsi formuler les théories des citations implicites, des apparences historiques, de la vérité relative de l'Écriture, des genres littéraires. Certes, ces auteurs se distinguent entre eux, tel un Loisy, un Lagrange, un Prat, un Hummelauer, par une diversité de méthodes et de positions, mais ils pratiquent tous l'exégèse critique, et ils appartiennent donc tous, du point de vue des gardiens de l'orthodoxie officielle, à l'école large.

D'où la longue série d'interventions du Saint-Office, de l'Index, de la Commission biblique, de la Consistoriale - les organismes de la Curie romaine étant contrôlés par les tenants de l'école étroite sous le pontificat de Pie X-qui tentent d'endiguer, voire de supprimer toute remise en question de l'exégèse scolastique et de ses principes directeurs, notamment en matière d'inerrance ${ }^{36}$. C'est un vrai "Combat pour la vérité de l'Écriture "37, pour reprendre le titre d'un ouvrage de l'un des coryphées du camp intransigeant, le jésuite Fonck, fondateur de l'Institut biblique pontifical en 1909, combat dont on trouvera une présentation synthétique dans l'article rédigé par Mangenot pour le Dictionnaire de théologie catholique ${ }^{38}$, ou dans les différentes entrées du Supplément du Dictionnaire de la Bible consacrées aux principes herméneutiques mentionnés ci-dessus. Mais on se souviendra que - comme nous l'avons indiqué au début- ces textes ont été rédigés après la publication, en 1920, de l'encyclique de Benoît XV et que leur reconstitution des positions des auteurs engagés dans le combat dépend de l'orthodoxie fixée par Spiritus paraclitus.

\section{L'encyclique Spiritus paraclitus}

Après la pause imposée par la Première guerre mondiale et dans le climat de détente qui marque le début du pontificat de Benoît XV, l'offensive antimoderniste, guidée en particulier par les cardinaux Merry del Val, secrétaire du Saint-Office, et van Rossum, président de la Commission biblique, reprend à la fin des années 1910, pour se prolonger dans les premières années du pontificat de Pie XI ${ }^{39}$. La conjoncture favorable aux tenants de l'école étroite semble leur avoir suggéré que l'heure était venue pour conclure définitivement le combat en leur faveur. L'opération, menée concrètement par le jésuite Alberto Vaccari, professeur d'Écriture sainte à l'Institut biblique pontifical, se

36 On trouvera la plupart de ces textes dans l'EB. Voir également les ouvrages de Beumer et de Burtchaell cités ci-dessus.

37 L. Fonck, Der Kampf um die Wahrheit der H. Schrift seit 25 Jahren, Innsbruck, Felizian Rauch, 1905.

38 Mangenot, op. cit., en particulier coll. 2237-2265.

39 É. Fouilloux, “Un regain d'antimodernisme? ”, P. Colin (éd.), Intellectuels chrétiens et esprit des années 20, Paris, Cerf, 1997, pp. 83-114, et, du même auteur, Une Église en quête de liberté, Paris, Desclée de Brouwer, 1998, pp. 15-26. 
fit en trois étapes : dénonciation publique des erreurs de 1'“ école large ", en 1919 ; proscription officielle dans l'encyclique Spiritus paraclitus, en 1920 ; élimination de l'un des principaux véhicules des opinions proscrites avec la mise à l'Index, en décembre 1923, de l'édition révisée par le sulpicien Brassac du Manuel biblique de Vigouroux.

Il est à relever qu'à l'enjeu théorique s'ajoute une rivalité entre centres de production intellectuelle: l'École biblique de Jérusalem, fondée et dirigée par le dominicain Lagrange, et l'Institut biblique pontifical, qui songe à ouvrir une succursale à Jérusalem, selon un projet du P. Fonck remontant à 1910, qui se réalisera en 192740 . L'entretien du P. Lagrange avec le cardinal van Rossum à ce sujet, en octobre 1918, montre bien l'irréductibilité des positions : car si, pour le cardinal, la Revue biblique n'adhère pas suffisamment à l'esprit des décrets du Saint-Siège, le dominicain refuse d'accepter les positions du " conservatorismo" en matière d'exégèse. D'où la réplique de van Rossum qui coupe court et salue la fondation de la succursale de l'Institut biblique à Jérusalem, comme institution qui suivra " la direction que donne l'Église "41.

Le feu est ouvert lors de la séance académique tenue en novembre 1918 à l'Institut biblique, lorsque l'orateur officiel, le P. Fonseca, s'en prend au modernisme, en incriminant également l'École biblique ${ }^{42}$. Bien plus important est le long article publié dans la Civiltà cattolica, entre novembre 1918 et février 1919, dans lequel Vaccari célèbre le 25e anniversaire de la publication de Providentissimus Deus, en rappelant toute l'importance de cette encyclique pour la formulation de la vraie doctrine catholique en matière d'inerrance biblique. Après avoir passé en revue les efforts entrepris par les catholiques en matière d'exégèse, le professeur de l'Institut biblique stigmatise toute une série de théories herméneutiques, déjà proscrites par différents décrets de la Commission biblique, mais que leurs auteurs s'efforcent de défendre grâce à une interprétation détournée de l'encyclique de Léon XIII. Vaccari identifie l'École biblique de Jérusalem comme centre du "parti qui s'efforce de concilier les enseignements de Léon XIII avec la critique moderne "43.

Le P. Lagrange, avec sa tentative, proposée dès la Méthode historique, d'étendre le

40 B. Montagnes, “Les séquelles de la crise moderniste: l'École biblique au lendemain de la Grande Guerre ", Revue thomiste, XC, 1990, 245-270, cité d'après le recueil d'études du même auteur, Le serviteur de Dieu Marie-Joseph Lagrange OP (1855-1939). Biographie critique, Rome, 1999, pp. $279 / 295$.

41 Entretien publié par F. Turvasi, Giovanni Genocchi e la controversia modernista, Roma, Edizioni di storia e letteratura, 1974, pp. 363-365, note 1.

42 Montagnes, Le serviteur, p. 277.

43 [A. Vaccari], “Venticinque anni dopo l'enciclica Providentissimus ", La Civiltà cattolica, 1918, t. 4, p. 361-374: 367 . 
critère exégétique des apparences, appliqué par l'encyclique de Léon XIII au domaine scientifique, également à l'histoire -ce qu'on appelait le critère des apparences historiques - est l'une des principales cibles de l'article. Tout aussi inacceptable est la théorie des genres littéraires, inaugurée - selon Vaccari-par Lenormant, et développée par Lagrange et par l'exégète jésuite Hummelauer, dont le principe fondateur, le fait de chercher le but de l'inspiration, et donc de l'enseignement de l'écrivain sacré, s'oppose formellement à l'enseignement de Providentissimus Deus. La doctrine de Loisy concernant la vérité relative de la Bible est également discutée mais son cas, en 1919, est déjà réglé44.

Dans un article de la Revue biblique de l'été 1919, Lagrange répond avec ce qu'il appellera une rétractation de son interprétation de Léon XIII ${ }^{45}$. Mais la prise de position officielle du magistère pontifical se préparait déjà. En transmettant un projet d'encyclique au cardinal van Rossum, en décembre 1919, le P. Fonck se plaint de la diffusion d'opinions contraires à Providentissimus Deus et aux décrets de la Commission biblique ${ }^{46}$. L'encyclique Spiritus paraclitus de Benoît XV, qui célèbre le 15e centenaire de la mort de saint Jérôme, est publiée fin septembre 1920.

La partie doctrinale de l'encyclique a été probablement rédigée par Fonck, peut-être avec la collaboration de Vaccari ${ }^{47}$. Dans tous les cas, les cinq pages de commentaire que ce dernier publie dans la Civiltà cattolica, immédiatement à la suite du texte de l'encyclique, pour en présenter les éléments essentiels, illustrent bien la dynamique de production d'orthodoxie formulée ci-dessus. Selon Vaccari, Benoît XV a voulu expliquer la pensée de Léon XIII en matière d'inerrance et rectifier les interprétations fausses qu'en donnaient de nombreux catholiques. Sont ainsi condamnés la distinction entre élément primaire et secondaire de l'Écriture, l'affirmation de sa vérité relative, le critère des apparences historiques, celui des citations implicites, celui des genres littéraires, c'est-à-dire tous les principes herméneutiques qui mettent en question " la stricte historicité des livres sacrés " 48 . Ces critères sont désormais à considérer comme

44 [A. Vaccari], “Venticinque anni dopo ”, La Civiltà cattolica, 1919, t. 1, p. 369 sq.

45 M.-J. Lagrange, “Addenda et notanda”, Revue Biblique, XVI(3-4), 1919, 593-600: 598. Cf. Montagnes, Le serviteur, p. 280.

46 Lettre citée par Turvasi, op. cit., p. 363, note 1.

47 Beumer, op. cit., p. 46. Dans les archives de la secrétairerie des lettres pontificales, on conserve quatre projets anonymes de l'encyclique, dont deux manuscrits et deux tapés à la machine, Archivio segreto vaticano, Epistolce ad principes, Positiones et minutce 164, dossier 1920, fascicule "Encyclica in Centenario S. Hieronymi”.

48 [A. Vaccari], “L'enciclica per il centenario di S. Girolamo ”, La Civiltà cattolica, 1920, t. 4, p. 42. Ce que soulignent également les “Observanda” qui précédent l'un des projets d'encyclique, tapé à la machine: "3. Capita doctrinæ quæ post Litteras Providentissimus Deus magis impugnata fuerunt, hæc 
proscrits et ne doivent plus être enseignés dans les écoles.

\section{L'affaire Brassac}

Après la dénonciation des erreurs et leur proscription officielle, restait la troisième étape, celle de l'application des disposition pontificales. Elle sera réalisée par la mise à l'Index de la quatorzième édition du Manuel biblique de Vigouroux, publiée dès 1917 par l'exégète sulpicien Brassac ${ }^{49}$. Pour expliquer cette mesure, qui devait susciter une grande surprise étant donné qu'elle frappait un ouvrage très prudent, le cardinal Merry del Val souligne, dans une lettre adressée au supérieur général de Saint-Sulpice, que l'ouvrage est en contradiction pour ce qui concerne " l'inspiration de la Sainte Écriture et son inerrance, surtout en matière historique", avec les Conciles, les documents romains récents et " la tradition catholique tout entière "50.

La longue série de citations que contient la lettre du cardinal secrétaire du SaintOffice est ouverte par celle du passage décisif de l'encyclique de Léon XIII — cité cidessus - qui condamne le principe doctrinal fondateur des théories herméneutiques réunies, surtout après l'article de Mgr d'Hulst, sous l'appellation d'école large : la causalité finale de l'inspiration biblique ${ }^{51}$. Merry del Val reproche à Brassac de "pencher vers les opinions de l'école large", de "détourner la signification [des documents du magistère ecclésiastique] dans le sens qui lui plaît personnellement", et d'avoir "complètement changé l'excellent esprit de l'ouvrage primitif de $\mathrm{M}$. Vigouroux "52. De son côté, le P. Lagrange reconnaissait que les volumes de l'édition Brassac concernant l'Ancien Testament “ ne ressemblaient guère à Vigouroux ”, et qu'il avait évité de publier l'analyse des différences pour ne pas compromettre l'ouvrage ${ }^{53}$.

Des explications plus développées seront formulées dans la Civiltà Cattolica, en juin 1924, de nouveau par Vaccari qui, à la même époque, est promu vice-recteur de l'Institut biblique et consulteur du Saint-Office. L'article de l'exégète jésuite illustre bien, une fois encore, le lien étroit qui subsiste entre doctrine de l'inspiration et interprétation de l'Écriture. Selon Vaccari, en dépit de la prétendue allégeance de Brassac au dogme de l'inspiration et de l'inerrance biblique, le sulpicien minimise

sunt, quæ in schemate pluribus evolvuntur: a) Restrictio inerrantiæ ad res religiosas. b) Omnimoda veritas historica narrationum biblicarum. c) Compositio Evangeliorum, præsertim quarti”, Archivio segreto vaticano, loc. cit.

49 Cf. Fouilloux, "Un regain d'antimodernisme?".

50 La Documentation catholique, XI, 1924, col. 324.

51 Ibid.

52 Ibid., col. 326sq.

53 Lettre du 25 janvier 1924, citée dans Montagnes, Le serviteur, p. 291. 
l'enseignement de Léon XIII, "magistralement interprété" par Benoît XV54. Non seulement les critères exégétiques qu'il adopte - distinction entre substance du récit et éléments accessoires, genres littéraires, apparences historiques - ont déjà été proscrits, mais encore "il est un principe qui forme la clef de voûte de tout le système, à savoir que le but religieux visé par l'auteur inspiré limite en proportion la valeur de ses affirmations. Les auteurs sacrés ne veulent rien enseigner que la vérité religieuse " ${ }^{55}$. Or ce principe a été réprouvé par les encycliques de Léon XIII et de Benoît XV56.

Vaccari conclut à la nécessaire proscription d'un manuel "où se trouvaient professées les théories spéciales à l'école large et à la nouvelle exégèse de certains catholiques "57. L'allusion au P. Lagrange, confirmée par le renvoi en note à l'article de la Civiltà cattolica de 1919, est transparente. De fait, on était conscient à l'époque que, derrière Brassac, c'était le P. Lagrange qui était visé, le sulpicien étant considéré comme " un disciple de l'École biblique ". Toutefois, grâce à la protection du Maître de l'Ordre et à l'intervention d'autres personnages influents, aucune mesure disciplinaire ne frappera l'institution dominicaine d'études bibliques ${ }^{58}$.

Une circulaire adressée par le Saint-Office aux supérieurs religieux, en mai 1924, impose aux professeurs d'exégèse de suivre les normes édictées par le Saint-Siège, en particulier dans les encycliques de Léon XIII et Benoît XV, et de s'y conformer dans le choix des manuels ${ }^{59}$. Vaccari et ses collègues de l'Institut biblique devaient bientôt publier un nouveau manuel d'exégèse en latin ${ }^{60}$, réédité plusieurs fois jusqu'au milieu du XXe siècle et destiné sans doute, dans leurs intentions, à prendre la place du Manuel biblique de Vigouroux et à affirmer définitivement leur conception de l'orthodoxie en matière d'exégèse. Dans l'introduction du premier volume, un collectif dirigé par Vaccari, l'exégète jésuite indique que ce manuel a été rédigé à la demande de la Congrégation des Études pour servir de base à la formation dans les séminaires ${ }^{61}$.

En fin observateur de la situation, Alfred Loisy écrivait à ce sujet, en 1925 :

Le livre [de Brassac], comme tel, au point de vue de la simple et stricte orthodoxie, n'a d'autre défaut que son honnêteté. [...] mais, pour détourner de Saint-Sulpice la clientèle d'élite qu'il avait su conserver, on a voulu ruiner son prestige en le rendant suspect

54 Traduction dans La Documentation catholique, XIII, 1925, coll. 420-430 : 422.

55 Ibid., col. 426.

56 Ibid., col. 428.

57 Ibid., col. 430 (souligné dans l'original).

58 Montagnes, Le serviteur, pp. 291-293.

59 Analecta Sacri Ordinis Fratrum Prcedicatorum, XXXII(4), 1924, 459-461. Cf. Montagnes, Le serviteur, p. 294.

60 Institutiones biblica scholis accomodatce, Rome, Institut biblique pontifical, 1925 sqq. 5e éd., Rome, Institut biblique pontifical, 1937 sqq.

61 Institutiones [...], t. I, Rome, Institut biblique pontifical, 1925, p. III. 
d'hétérodoxie sur un point délicat [...]: 1'interprétation des Écritures. Saint-Sulpice n'avait pas su garder comme il faut la source de la foi ! À qui s'adresser maintenant? À la capitale infaillible de l'orthodoxie. C'est vers Rome évidemment que les clercs soucieux de la bonne théologie, et aussi de leur avenir en ce monde, devront se diriger : là [...] ils seront initiés à la saine doctrine en suivant les cours des jésuites au Collège romain ${ }^{62}$.

Analyse lucide de la situation du champ théologique, et des luttes pour l'appropriation $\mathrm{du}$ capital symbolique, après la deuxième intervention importante du magistère pontifical en matière d'inspiration et d'herméneutique biblique.

\section{Conclusion}

Arrivés à ce point, il faudrait étudier la réception de cette nouvelle orthodoxie, de même que les détours inaugurés par les agents appartenant au camp adverse pour continuer à pratiquer l'exégèse critique. Mais ce n'est pas le lieu ici. Je reviens donc à ma question du départ : comment mesurer l'orthodoxie d'un auteur? Loisy était-il orthodoxe ? Pouvait-il l'être étant donné l'imposition de la nouvelle orthodoxie par les encycliques de Léon XIII et Benoît XV ? La réponse à ces questions est, évidemment, négative, et elle vaut d'ailleurs tout autant pour Loisy, que pour Lagrange et Brassac. Ce n'est que l'insoumission des auteurs de l'école large, dans leur pratique exégétique, à l'orthodoxie imposée officiellement par l'école romaine qui permettra la modernisation de l'exégèse catholique et une reconfiguration du champ théologique qui sera officiellement reconnue, non sans combats, au Concile Vatican II ${ }^{63}$.

Pour Alfred Loisy, on le sait, le chemin fut différent. En conclusion, c'est à lui que je laisserai la parole, en citant un récit autobiographique rédigé en 1936. En retraçant les débuts de son parcours intellectuel, Loisy écrit :

Renan m'apprit à discuter scientifiquement les textes de la Bible, et ce que j'inférai de ses leçons fut que le commentaire scientifique de l'Écriture était à refondre entièrement dans l'Église catholique pour s'adapter aux conditions réelles de la culture de notre temps.

Je ne m'avisais pas d'abord que cette conclusion ruinait en principe toute l'économie de la tradition théologique dans l'Église romaine, et qu'elle compromettait à fond certains dogmes; [...] Il était impossible que je ne prisse pas bientôt moi-même une conscience nette de la radicale incompatibilité de mes conclusions générales, fondées sur l'expérience critique, avec l'orthodoxie romaine ${ }^{64}$.

62 A. Loisy, L'Église et la France, Paris, Emile Nourry, 1925, pp. 156-157. Je remercie Pierre Colin d'avoir attiré mon attention sur cet ouvrage.

63 R. Burigana, La Bibbia nel concilio, Bologna, Il Mulino, 1998.

64 Texte publié par É. Poulat, Critique et mystique, Paris, Le Centurion, 1984, pp. 14-43 : 19-20. 